
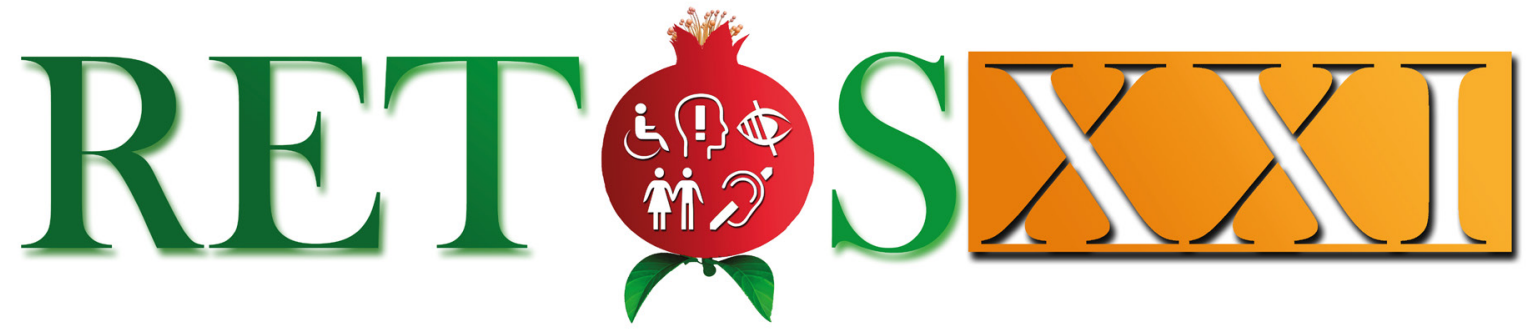

LA NECESIDAD DE INVESTIGACIÓN-ACCIÓN COMO MEJORA EDUCATIVA THE NEED FOR RESEARCH-ACTION AS AN EDUCATIONAL IMPROVEMENT

\title{
ESTUDIO DE LA COMPETENCIA LECTORA Y ESCRITORA DE ALUMNOS CON DISCAPACIDAD DE LA PROVINCIA DE HERRERA (PANAMÁ)
}

\section{STUDY OF THE READING AND WRITING COMPETENCE OF STUDENTS WITH DISABILITIES IN THE PROVINCE OF HERRERA (PANAMÁ}

Ferdinan Pérez Vega , Eriberto Calderón

Volumen 3, 2019.

Enviado: 29/4/2019

Aceptado: $2 / 5 / 2019$ 


\section{ESTUDIO DE LA COMPETENCIA LECTORA Y ESCRITORA DE ALUMNOS CON DISCAPACIDAD DE LA PROVINCIA DE HERRERA (PANAMÁ)}

\section{STUDY OF THE READING AND WRITING COMPETENCE OF STUDENTS WITH DISABILITIES IN THE PROVINCE OF HERRERA (PANAMÁ)}

Ferdinan Pérez Vega1, Eriberto Calderón²

1 Licdo. Educación Especial. Instituto Panameño de Habilitación Especial (IPHE). fer2913@hotmail.com.

2 Licdo. Educación Especial. Instituto Panameño de Habilitación Especial (IPHE). eriberto221006@gmail.com 


\section{Resumen}

La lectura y la escritura no solo las podemos definir como herramientas académicas sino como procesos básicos de comunicación inherente a la vida personal y social de los seres humanos. Esta es la justificación para evaluar tales competencias en el alumnado de la provincia de Herrera (Panamá). Concretamente, su desarrollo del lenguaje, en sus dimensiones básicas de forma, contenido y uso, mediante la prueba PLON-R como base para la adquisición del lenguaje escrito. Y seguidamente se aplican las pruebas del PROLEC-R y del PROESC para la lectura y la escritura, respectivamente. Todas ellas como parte de nuestra investigación en la provincia de Herrera en los niveles de primaria y premedia, la cual tiene como objetivo la evaluación de tales competencias de cara a detectar dificultades en sendas etapas para mejorar la calidad lectora y de escritura de nuestros estudiantes. Así se han explicitado en este artículo las deficiencias orales, lectoras y escritoras de acuerdo con los programas anteriores, y se han propuesto sugerencias de mejora contextualizadas y personalizadas, dado que tanto la evaluación como la intervención han sido llevadas a cabo por los propios docentes de los alumnos, en lo que cabría denominarse Investigación-Acción.

Palabras clave: competencia comunicativa, lectura, escritura, aprendizaje del lenguaje escrito y desarrollo, dificultades y deficiencias comunicativas.
Reading and writing can not only be described as academic tools but as a basic communication process that is inherent in the personal and social life of human beings. This is the justification for evaluating such competences in the students of the province of Herrera (Panamá). Specifically, its development of language, in its basic dimensions of form, content and use, through the PLON-R test as a basis for the acquisition of written language. And then the PROLEC-R and PROESC tests for reading and writing are applied respectively. All of them as part of our research in the province of Herrera in the levels of elementary and junior high schools, which aims to evaluate such skills in order to detect difficulties in both stages to improve the reading and writing qualities of our students. In this way, speaking, reading and writing deficiencies, in accordance with the previous programs, have been explained in this article, and suggestions for contextualized and personalized improvements have been proposed, considering that both the research and the intervention have been carried out

by the teachers themselves, in what could be called Research-Action.

Keyword: communicative competence, reading, writing, written language learning, development, communicative difficulties and deficiencies. 


\section{INTRODUCCIÓN}

Enesteartículo seanalizan, desde los modelos cognitivos de lectura y escritura (Aaron, 1990, y Hayes y Flower, 1980, respectivamente), el aprendizaje y desarrollo de la lectoescritura de los estudiantes con discapacidad intelectual de la provincia de Herrera, para lo cual se les aplicó diferentes pruebas como PLON-R, PROLEC-R, PROESC, debidamente detallados en sus publicaciones correspondientes, al tratarse de programas estandarizados: Aguinaga, Armentia, Fraile, Olangua y Uriz (2004), Cuetos, Rodríguez, Ruano y Arribas (2014), Cuetos, Ramos, y Ruano (2004), respectivamente.

Se han desarrollado multitud de investigaciones sobre la evaluación de la lectura y escritura, en algunos contextos está institucionalizado, como evaluación propia del sistema educativo. Sin duda las más afines a esta son las que se presentan en el monográfico dela revista PINCEDUC I+D (número 2), dedicado a la investigación sobre alfabetización de la población escolar con discapacidades desarrollada en el ámbito de la República de Panamá, durante los años 2016 al 2018, por el Dr. Rodríguez en colaboración con la Red de Investigadores del Instituto Panameño de Habilitación Especial (IPHE).

Concretamente, la investigación global que describe la situación nacional competencial del alumnado con todas las discapacidades posibles de primaria y premedia de Panamá (Rodríguez, 2019). Así como también las evaluaciones distribuidas por regiones o provincias, como la de Atkins y Alveo (2019) llevada a cabo en la ciudad de Panamá (Panamá), la de De Cerrud, Bermúdez, Barrett, Santana y González (2019) desarrollada en el ámbito de Coclé (Panamá), la de Orozco (2019) para el ámbito de Panamá Este (Panamá), la de Pérez, De Acosta y Rodríguez (2019) desarrollada en Darién (Panamá), y, por último por orden alfabético y no de importancia, la de Swaby (2019) en el contexto de Panamá Oeste (Panamá).

Tales investigaciones, complementarias de la que se presenta en esta ocasión para el ámbito concreto de Herrera, se constituyen a la vez en referentes de esta. En todas ellas pueden apreciarse las diferentes dificultades que la población escolar con discapacidad ha de afrontar en el sistema educativo panameño como consecuencia de la deficiencia en el input de información del exterior, así como posibles soluciones propuestas desde las reflexiones y capacitaciones ofrecidas por el IPHE en el seno de sus instalaciones y bajo el auspicio de su financiación.

Sirviendoelanteriorenunciadocomoproblema de la investigación, como objetivo principal de investigación se propone conocer con precisión el grado de dominio y las posibles dificultades que presentan los alumnos con discapacidades de los niveles últimos de primaria y primeros de premedia de la provincia de Herrera en las tareas de lectura y escritura, habilidades instrumentales básicas. Más concretamente, estas pruebas nos ayudan a visualizar cuál es el desarrollo lingüístico, análisis de lectura, redacción, ortografía y signos de puntuación, entre otros. De este objetivo principal o finalidad básica se desprenden los siguientes objetivos específicos u operativos:

- Descubrir si los alumnos de la muestra representativa de la población de Herrera muestran un dominio básico de lenguaje oral, correspondiente con los niveles de enseñanzas básicas infantiles, para lo cual se empleará la herramienta del PLON-R.

- Conocer tanto el grado de dominio como las dificultades del alumnado con discapacidad escolarizados en escuelas de Herrera, mediante el empleo del programa de evaluación lectora PROLEC-R.

- Descubrir igualmente el dominio y dificultades en el mismo alumnado, pero con respecto de su expresión escrita, valiéndonos para ello del programa específico PROESC.

- Establecer diferencias (por ejemplo, entre sexo o etapa) y relaciones (entre lenguaje 
oral, lectura y escritura) entre los datos obtenidos con los programas anteriores que arrojen luz sobre la adquisición y optimización lectoescritora.

- Proponer estrategias y recursos concretos para fortalecer la competencia lectoescritora en base a los resultados de las pruebas anteriores, fomentando el hábito de lectura empezado desde los primeros grados de primaria, estimulando a los estudiantes de la provincia de Herrera con estos hábitos de lectoescritura y la utilización de material tecnológico.

\section{MÉTODO DE LA INVESTIGACIÓN}

Dentro del diseño de investigación del Estudio de Casos múltiple seguido para este proceso de indagación, se ha procurado la recogida de información con objeto de conocer y mejorar la realidad, aunque tal mejora se acometerá en un segundo momento, de ahí que no alcance la denominación de diseño de InvestigaciónAcción.

\section{Recogida de datos. Instrumento}

De manera que, en primer lugar, se precisa la recogida de datos relevantes y con exactitud sobre la competencia manifiesta en el dominio de la lengua oral y la lectoescritura. Para tal recogida, se han empleado los programas arriba aludidos y abajo referenciados de los autores: el de Aguinaga, Armentia, Fraile, Olangua y Uriz (2004) para la evaluación de la lengua oral, el de Cuetos, Rodríguez, Ruano y Arribas (2014) para la evaluación de la competencia lectora, y el de Cuetos, Ramos y Ruano (2004) para la evaluación de la composición escrita.

Esto propiciará, en su segundo momento, establecer algunos métodos de aprendizajes que puedan implementar con los estudiantes de la provincia de Herrera para un buen desarrollo académico del lenguaje y la comunicación escrita y su incidencia en el resto de los aprendizajes. Tales mejoras serán aplicadas de forma contextualiza con los alumnos estudiados, tras lo que se acometerán nuevos procesos de evaluación que activarán de nuevo el proceso antes descrito.

\section{Participantes del estudio}

Respecto a la descripción de los participantes, las pruebas han sido aplicadas a estudiantes de nivel primario (IV, V y VI grado) y nivel secundario (IX grado), siete en total. Cabe señalar que estas pruebas se realizaron en tres centros educativos de la provincia de Herrera (Panamá), a cuatro estudiantes del nivel primario en el distrito de Chitré y a tres estudiantes del nivel secundario en el nivel de premedia del distrito de Ocú, cuyas características intrínsecas pueden observarse a continuación (ver tabla 1).

\section{Tabla 1. Características identitarias del alumnado de la muestra de estudio}

\begin{tabular}{|l|l|l|l|l|l|l|}
\hline Alumno & Escuela & Discapacidad & Edad & Etapa & Curso & Sexo \\
\hline L. M. & $\begin{array}{l}\text { Juan T del } \\
\text { Busto }\end{array}$ & Intelectual & 14 & 1 & $6^{\circ}$ & $\mathrm{M}$ \\
\hline Z. C. & $\begin{array}{l}\text { Juan T del } \\
\text { Busto }\end{array}$ & Intelectual & 10 & 1 & $5^{\circ}$ & $\mathrm{F}$ \\
\hline J. M. & $\begin{array}{l}\text { Eneida de } \\
\text { Castillero }\end{array}$ & Intelectual & 9 & 1 & $4^{\circ}$ & $\mathrm{M}$ \\
\hline Á. V. & $\begin{array}{l}\text { Eneida de } \\
\text { Castillero }\end{array}$ & Intelectual & 13 & 1 & $6^{\circ}$ & $\mathrm{M}$ \\
\hline A. B. & Rafael Quintero & Intelectual & 14 & 2 & $9^{\circ}$ & $\mathrm{M}$ \\
\hline B. O. & Rafael Quintero & Intelectual & 15 & 2 & $9^{\circ}$ & $\mathrm{M}$ \\
\hline R. S. & Rafael Quintero & Intelectual & 16 & 2 & $9^{\circ}$ & $\mathrm{M}$ \\
\hline
\end{tabular}

Fuente: Elaboración propia a partir de los datos recopilados en las pruebas. 


\section{RESULTADOS DE INVESTIGACIÓN}

A continuación, se muestran los resultados obtenidos, comenzando con el lenguaje oral y continuando con la lectura y escritura, dimensión cada una correspondiente con cada uno de los programas aplicados.

Resultados sobre la competencia lingüística oral (plon-r)

Según los datos recogidos en la tabla 2, los alumnos investigados no tienen problemas en el lenguaje oral, en ninguna de sus dimensiones, mostrando un dominio "normal (N)" del mismo. Salvo dos de ellos (28.57\%) que muestran dificultades en la dimensión forma del lenguaje, donde muestra una puntuación baja correspondiente con la valoración "necesita mejorar (NM)".

Tabla 2. Resultados particulares de la aplicación del programa PLON-R

\begin{tabular}{|c|c|c|c|c|c|c|c|c|}
\hline AREA & $\mathbf{1}$ & $\mathbf{2}$ & $\mathbf{3}$ & $\mathbf{4}$ & $\mathbf{5}$ & $\mathbf{6}$ & $\mathbf{7}$ & Media \\
\hline FORMA & $2.5 /$ & $2.5 /$ & $2.25 /$ & $2.5 /$ & $2.5 / \mathrm{N}$ & $2 / \mathrm{NM}$ & $2.5 / \mathrm{N}$ & $\begin{array}{c}2.39 / \\
\mathrm{N}\end{array}$ \\
\hline CONTENIDO & $\begin{array}{c}4.5 / \\
\mathrm{N}\end{array}$ & $\begin{array}{c}4.5 / \\
\mathrm{N}\end{array}$ & $4.5 / \mathrm{N}$ & $\begin{array}{c}4.5 / \\
\mathrm{N}\end{array}$ & $5 / \mathrm{N}$ & $4.5 / \mathrm{N}$ & $5 / \mathrm{N}$ & $\begin{array}{c}4.64 / \\
\mathrm{N}\end{array}$ \\
\hline USO & $6 / \mathrm{N}$ & $6 / \mathrm{N}$ & $5 / \mathrm{N}$ & $6 / \mathrm{N}$ & $5 / \mathrm{N}$ & $5 / \mathrm{N}$ & $6 / \mathrm{N}$ & $\begin{array}{c}5.57 / \\
\mathrm{N}\end{array}$ \\
\hline TOTAL & $13 /$ & $\begin{array}{c}13 / \\
\mathrm{N}\end{array}$ & $\begin{array}{c}12.75 / \\
\mathrm{N}\end{array}$ & $13 \mathrm{~N}$ & $\begin{array}{c}12.5 / \\
\mathrm{N}\end{array}$ & $\begin{array}{c}11.5 / \\
\mathrm{N}\end{array}$ & $\begin{array}{c}13.5 / \\
\mathrm{N}\end{array}$ & $\begin{array}{c}12.75 / \\
\mathrm{N}\end{array}$ \\
\hline
\end{tabular}

Fuente: elaboración propia.

Resultados sobre la competencia lectora y dificultades (prolec-r)

De los resultados obtenidos con el PROLEC-R se infiere la baja competencia lectora de los alumnos estudiados, como puede observarse en la tabla anterior, el $100 \%$ de los alumnos muestra una puntuación de "dificultades severas (DD)". El detalle de esta aseveración puede observarse en la tabla y leerse a continuación: 
Tabla 3. Resultados particulares de la aplicación del programa PROLEC-R $R^{3}$

\begin{tabular}{|c|c|c|c|c|c|c|c|c|}
\hline Categorías & 1 & 2 & 3 & 4 & 5 & 6 & 7 & Moda $^{4}$ \\
\hline \multirow[t]{2}{*}{ Nombre de letras } & 30 & 32 & 17 & 15 & 8 & 33 & 26 & $100 \%$ \\
\hline & $\mathrm{DD}$ & DD & DD & DD & DD & DD & DD & DD \\
\hline \multirow[t]{3}{*}{ Igual diferente } & 9 & 15 & 8 & 15 & 15 & 15 & 14 & 71.43 \\
\hline & DD & D & $\mathrm{D}$ & $\mathrm{D}$ & $\mathrm{D}$ & DD & $D$ & $\%$ \\
\hline & & & & & & & & D \\
\hline \multirow[t]{2}{*}{ Lectura de palabras } & 49 & 32 & 27 & 40 & 32 & 29 & 34 & $100 \%$ \\
\hline & DD & DD & DD & DD & DD & DD & DD & DD \\
\hline \multirow{3}{*}{$\begin{array}{ll}\text { Lectura } & \text { Pseudo } \\
\text { palabras } & \end{array}$} & 38 & 34 & 20 & 33 & 31 & 30 & 37 & 71.43 \\
\hline & D & D & $\mathrm{D}$ & DD & $\mathrm{D}$ & DD & $D$ & $\%$ \\
\hline & & & & & & & & D \\
\hline \multirow{3}{*}{$\begin{array}{l}\text { Estructuras } \\
\text { gramaticales }\end{array}$} & 14 & 14 & 15 & 14 & 13 & 12 & 14 & 57.14 \\
\hline & $\mathrm{N}$ & $N$ & $\mathrm{~N}$ & $N$ & $\mathrm{D}$ & $\mathrm{D}$ & $\mathrm{D}$ & $\%$ \\
\hline & & & & & & & & $\mathrm{N}$ \\
\hline \multirow[t]{2}{*}{ Signos de Puntuación } & 0 & 8 & 9 & 8 & 10 & 12 & 13 & $100 \%$ \\
\hline & DD & DD & DD & DD & DD & DD & DD & DD \\
\hline \multirow{2}{*}{$\begin{array}{l}\text { Comprensión } \\
\text { oraciones }\end{array}$} & 14 & 14 & 16 & 16 & 15 & 13 & 14 & 57.14 \\
\hline & D & D & $\mathrm{N}$ & $\mathrm{N}$ & $\mathrm{D}$ & DD & $\mathrm{D}$ & $\% \mathrm{D}$ \\
\hline \multirow[t]{2}{*}{ Comprensión Textos } & 12 & 10 & 3 & 9 & 8 & 9 & 10 & 57.14 \\
\hline & $\mathrm{N}$ & $\mathrm{N}$ & $\mathrm{D}$ & D & $\mathrm{D}$ & $\mathrm{D}$ & $\mathrm{N}$ & $\% \mathrm{D}$ \\
\hline \multirow[t]{2}{*}{ Comprensión Oral } & 6 & 2 & 2 & 4 & 5 & 5 & 6 & 57.14 \\
\hline & $\mathrm{N}$ & D & $\mathrm{D}$ & $N$ & $\mathrm{~N}$ & $\mathrm{D}$ & $\mathrm{N}$ & $\% N$ \\
\hline
\end{tabular}

${ }^{3}$ Valoraciones posibles: $\mathrm{DD}=$ Dificultad Grave, $\mathrm{D}=$ Dificultad leve, $\mathrm{N}=$ Normal

${ }^{4} \mathrm{Se}$ ha empleado la moda en lugar de la media como medida de tendencia central, puesto que al ser las puntaciones baremadas por edades y curso escolar la media no aporta ningún valor categorial.

\begin{tabular}{|c|c|c|c|c|c|c|c|c|}
\hline Categorías & $\mathbf{1}$ & $\mathbf{2}$ & $\mathbf{3}$ & $\mathbf{4}$ & $\mathbf{5}$ & $\mathbf{6}$ & $\mathbf{7}$ & Moda $^{4}$ \\
\hline TOTAL & DD & DD & DD & DD & DD & DD & DD & DD \\
& 161 & 159 & 128 & 150 & 137 & 158 & 168 & $100 \%$ \\
\hline
\end{tabular}

Fuente: elaboración propia. 
- En la primera de las categorías evaluadas por el programa, el nombre de las letras, el $100 \%$ presenta una "dificultad severa (DD)".

- En la categoría de igual y diferente la mayoría $(71.43 \%)$ muestra "dificultades leves (D)" frente al resto (28.57\%) que las presenta "severas (DD)".

- La dimensión de lectura de palabras aparece claramente deficiente, dada la unanimidad $(100 \%)$ de puntuaciones correspondientes con "dificultades severas (DD)", lo que denota mal uso de la ruta visual.

- Ligeramente mejor se observa la ruta auditiva, puesto que la competencia mostrada es mejor, aunque sigue siendo deficiente, dada la prevalencia de las "dificultades leves (D)" en un $71.43 \%$ de la muestra y aumenta la preocupación de ella con el resto que muestra "dificultades severas (DD)".

- La estructura gramatical muestra que el $57.14 \%$ presentan dificultades leves (D) y un $43 \%$ tiene un desarrollo normal.
- En los signos de puntuación un 100\% tiene dificultad severa.

- En comprensión de oraciones el $57.7 \%$ tiene "dificultad leve (D)" y un $43 \%$ no presentan dificultades sino competencia "normal (N)".

- En la comprensión de textos se reflejan los resultados iguales a la comprensión de oraciones.

- La comprensión oral refleja que un 57.14 $\%$ tienen "dificultades leves (D)", mientras que un $43 \%$ no presentan dificultades sino competencia "normal $(\mathrm{N})$ ".

\section{Resultados sobre la competencia escritora $y$ dificultades (PROESC)}

Los resultados generales de la provincia de Herrera en cuanto al PROESC, tras su contraste con la baremación del mismo de acuerdo con sus edades, reflejan que en la competencia escrita todos los participantes presentan "deficiencias severas (DD)", en la mayoría de las dimensiones, tal y como se aprecia en la tabla 4 y se comenta a continuación: 
Tabla 4. Resultados particulares de la aplicación del programa PROESC ${ }^{5}$

\begin{tabular}{|c|c|c|c|c|c|c|c|c|}
\hline AREAS & 1 & 2 & 3 & 4 & 5 & 6 & 7 & Moda \\
\hline \multirow[t]{2}{*}{ Dictado de silaba } & DD & $\overline{D D}$ & $\overline{D D}$ & DD & $\overline{D D}$ & $\overline{D D}$ & $\overline{D D}$ & $100 \%$ \\
\hline & 17 & 18 & 16 & 19 & 21 & 19 & 20 & DD \\
\hline \multirow[t]{2}{*}{ Ortografía Arbitraria } & DD & $\overline{D D}$ & $\mathrm{D}$ & DD & DD & $\mathrm{DD}$ & $\overline{D D}$ & $85 \%$ \\
\hline & 15 & 18 & 14 & 14 & 17 & 13 & 19 & DD \\
\hline \multirow[t]{2}{*}{ Ortografía reglada } & DD & $\mathrm{D}$ & DD & DD & DD & $\overline{D D}$ & DD & $85 \%$ \\
\hline & 15 & 17 & 14 & 16 & 19 & 17 & 19 & DD \\
\hline \multirow{2}{*}{$\begin{array}{l}\text { Dictado } \\
\text { pseudopalabras }\end{array}$} & DD & DD & $\overline{D D}$ & DD & $\overline{D D}$ & $\overline{D D}$ & DD & $100 \%$ \\
\hline & 15 & 18 & 17 & 14 & 18 & 15 & 20 & DD \\
\hline \multirow{2}{*}{$\begin{array}{l}\text { Pseudopalabras } \\
\text { Reglas ortográficas }\end{array}$} & DD & $\overline{D D}$ & DD & DD & $\overline{D D}$ & $\overline{D D}$ & DD & $100 \%$ \\
\hline & 6 & 7 & 5 & 7 & 9 & 5 & 8 & DD \\
\hline \multirow{2}{*}{$\begin{array}{lll}\text { Dictado } & \text { de } & \text { frases } \\
\text { (acento) } & & \\
\end{array}$} & DD & NB & $\mathrm{DD}$ & DD & $\overline{D D}$ & $\mathrm{DD}$ & $\overline{D D}$ & $85,7 \%$ \\
\hline & 1 & 3 & 1 & 1 & 2 & 1 & 3 & DD \\
\hline \multirow{2}{*}{$\begin{array}{l}\text { Dictado de frases } \\
\text { (mayúscula) }\end{array}$} & DD & DD & DD & DD & DD & DD & DD & $100 \%$ \\
\hline & 4 & 6 & 2 & 4 & 8 & 3 & 6 & DD \\
\hline \multirow[t]{2}{*}{ Signos De puntuación } & DD & DD & DD & DD & $\mathrm{DD}$ & $\mathrm{DD}$ & DD & $100 \%$ \\
\hline & 2 & 3 & 1 & 2 & 3 & 1 & 3 & DD \\
\hline \multirow[t]{2}{*}{ Escritura de un cuento } & NB & NB & $\overline{N B}$ & NB & NB & NB & NB & $100 \%$ \\
\hline & 5 & 6 & 5 & 5 & 5 & 6 & 5 & NB \\
\hline \multirow{2}{*}{$\begin{array}{lll}\text { Escritura de } & \text { una } \\
\text { redacción } & & \\
\end{array}$} & NB & NB & NB & NB & NB & NB & NB & $100 \%$ \\
\hline & 5 & 5 & 5 & 6 & 5 & 5 & 6 & NB \\
\hline \multirow[t]{2}{*}{ TOTAL } & DD & DD & DD & DD & DD & $\mathrm{DD}(85)$ & DD & DD \\
\hline & (87) & (103) & (86) & (90) & (112) & & (114) & $100 \%$ \\
\hline
\end{tabular}

Fuente: Elaboración propia.

\footnotetext{
${ }^{5}$ Valoraciones posibles: $\mathrm{DD}=$ Dificultad Grave, $\mathrm{D}=$ Dificultad Leve, $\mathrm{NB}=$ Normal Bajo, $\mathrm{NM}=$ Normal Medio, $\mathrm{NA}=$ Normal Alto.

- En la competencia mostrada en el dictado de sílabas, el $100 \%$ presenta "dificultad severa (DD)".

- En los ejercicios sobre ortografía arbitraria un $85 \%$ presenta "dificultad severa (DD"),

mientras que uno de ellos (15\%) refleja "dificultad leve (D)".

- Idéntico porcentaje se manifiesta en la ortografía reglada: el $85 \%$ refleja "dificultad severa (DD)" mientas que apenas el $15 \%$ presenta "dificultad leve (D)".
} 
- El uso de las reglas ortográficas se muestra deficiente: el 100\% muestra "dificultad severa (DD)".

- Respecto del uso del acento, el $85,71 \%$ presenta "dificultad severa (DD)", mientras el $28.6 \%$ refleja una competencia "normal (N)".

- El empleo de las letras mayúscula por su propia naturaleza o bien por el lugar de la frase que ocupen ha resultado complicado para los alumnos, el $100 \%$ refleja "dificultad severa (DD)".

- De la misma manera que el empleo de los signos de puntuación: el $100 \%$ refleja "dificultad severa (DD)".

- Dada la valoración subjetiva que proporciona la prueba respecto de la escritura de un cuento, los alumnos han mostrado en su totalidad (100\%) una "competencia normal baja (NB)".

- E igual ha acontecido con respecto a la escrituradeunaredacción, evidenciándose en el $100 \%$ de los participantes los indicadores proporcionados por el programa para apuntar hacia una competencia "normal con tendencia a baja (NB)".

Resultados globales de las tres dimensiones del lenguaje evaluadas

Cabe resaltar el tratamiento estadístico de los datos presentados en la tabla siguiente (ver tabla 5) a través del cálculo de la correlación entre lenguaje oral, lectura y escritura, mediante la prueba no paramétrica rho de Spearman, que los alumnos que han obtenido mejores puntaciones en el lenguaje oral son a su vez los que mejores han obtenido en la lectura, aunque no de manera muy intensa $(r=0.48)$ pero sí de manera significativa $(p=0.04)$, y que ninguna de ellas relaciona con la escritura. En este caso, viene a indicar que la buena adquisición del lenguaje oral contribuye a un mejor desarrollo de la lectura, pero no así respecto de la escritura; como tampoco el dominio lector correlaciones con el dominio escritor

Tabla 5. Correlaciones entre puntuaciones en las distintas pruebas

\begin{tabular}{|c|c|c|c|}
\hline & TOTAL PLON & TOTAL PROLEC-R & TOTAL PROESC \\
\hline TOTAL PLON & 1 & & \\
\hline$N$ & - & & \\
\hline$P$ & - & & \\
\hline TOTAL PROLEC-R & .48 & 1 & \\
\hline$N$ & 7 & - & \\
\hline$P$ & .04 & - & \\
\hline \multirow[t]{3}{*}{ TOTAL PROESC } & .174 & .124 & 1 \\
\hline & 7 & 7 & $N$ \\
\hline & .71 & .79 & $P$ \\
\hline
\end{tabular}

Fuente: Elaboración propia. 


\section{CONCLUSIONES Y PROYECCIÓN DIDÁCTICA}

Ha de concluirse en primer lugar que ha sido posible verificar el dominio lectoescritor de alumnos con discapacidades para conocer el punto de partida de la intervención necesitada, objeto principal de este estudio. Dadas las características de los participantes, no se pretende generalizar estos resultados sino acometer acciones educativas ajustadas a los escenarios encontrados. Desde este prisma, lo cierto es que se han observado niveles bajo de dominio lector y aún más escritor, a diferencia del lenguaje oral, que se encuentra en un nivel de adquisición y desarrollo normal. En esta primera conclusión que se detalla a continuación no se hallan diferencias respecto de los estudios similares analizados para la situación del estado de la cuestión (De Cerrud, Bermúdez, Barrett, Santana \& González, 2019; Orozco, 2019; Pérez, De Acosta \& Rodríguez, 2019; Rodríguez, 2019; Swaby, 2019).

En concreto, unas de las debilidades que se observa con mayor frecuencia en la provincia de Herrera en la falta de compresión lectora. Complementariamente, las dificultades en la expresión escrita se hacen evidentes en todas las dimensiones evaluadas por el programa. Ello es importante en la formación educativa de nuestros centros escolares para que puedan desarrollar sus habilidades en la lectoescritura.

La lectoescritura es de suma importancia ya que les ayuda en su rendimiento académico, social y su vida cotidiana. Es importante señalar que la lectoescritura ayuda de manera permanente a nuestros estudiantes en la comprensión lectora y por medio de los aprendizaje que el lector interactúa con sus análisis y objeto para favorecer la comprensión.

Para mejorar la lectoescritura en nuestra provincia es importante aumentar la carga lectiva de la asignatura de español, utilizando materiales concretos como rompecabezas de oraciones, realizar carteles, usar software de lectura y escritura, leer cuentos y hacer rincones interactivos de lectoescritura. En concreto se presentan a continuación, como ilustración, algunas estrategias para optimizar la competencia lingüística de estos alumnos, aunque también se pueda acudir a programas ya estandarizados, como el de Lecuona (1999), o propuestas concretas, como las explicitadas por Treviño, Pedroza, Pérez, Ramírez, Ramos y Treviño (2007), y emplear de esas obras las actividades de las dimensiones resaltadas, es decir, las afectadas en el alumnado:

- Sugerencias para mejorar el lenguaje oral de los alumnos. Pueden utilizarse recursos como el cuento, con objeto de imitar sonidos naturales como el del viento, las olas del mar, piedras cayendo, lluvia, entre otros

- Estrategias didácticas para mejorar la lectura en las dimensiones más afectadas. Por ejemplo, para interiorizar el nombre de letras emplear la pronunciación de la letra $\mathbf{A}$ según el sonido (una persona asustada grita aaaaaaaa...). Respecto a la diferenciación entre letras similares pero no iguales puede recurrirse a la $\tilde{\mathbf{N}}$ identificada como el sonido de un llanto de un bebé. Para la lectura de palabras identificar las palabras desconocidas mediante la decodificación. Con ello se trabaja también la lectura de pseudopalabras, realizando también diversos tipos de juegos como el (veo-veo), para ir encadenando palabras, juegos con tarjetas de letras. Se requiere mejorar el empleo de los signos de puntuación, lo cual se puede trabajar con libros de cuento con animales, de la naturaleza, medios de transporte y comunicación, a través de una lectura acompañada entre docente y alumnado, destacando a medida que se desarrolla el uso de los signos de puntuación que reconozcan y lo nuevos, enfatizando en todos su pronunciación. Para la comprensión de oraciones conviene facilitar al alumno listas de recetas, como por ejemplo de un restaurante, para iniciar la comprensión de lo que dice el texto debido a que están compuestas por frases cortas. Complementario, para la comprensión de textos la técnica del subrayado contribuye a incrementar la comprensión de los principales puntos y detalles de un texto.

- Estrategias didácticas para optimizar la escritura en todas sus dimensiones, dado que es la competencia menos desarrollada, Por ejemplo, para mejorar el resultado en los dictados de palabras y frases se 
sugiere dictados de palabras de la misma familia y se construyen frases con ellas. Respecto de la mejora competencial de la ortografía y de los signos de puntuación se ofrece la estrategia de trabajo mediante los cuentos, adivinanzas y fábulas para que los estudiantes lean después.

\section{REFERENCIAS}

Aguinaga, G., Armentia, M. L., Fraile, A., Olangua, P., \& Uriz, M. (2004). PLON-R. Prueba de Lenguaje Oral, Revisada. Madrid: TEA

Atkins, D. E., \& Alveo, L. (2019). Estudio descriptivo de las competencias en lectura y escritura de la población escolar del Instituto Panameño de Habilitación Especial, Panamá Centro. Revista Pinceduc I+D, 2, 143-150.

Cuetos, F., \& Valle, F. (1988). Modelos de lectura y dislexias. Infancia y Aprendizaje, 11(44), 3-19.

Cuetos, F., Rodríguez, B., Ruano, E., \& Arribas, D. (2014). "PROLEC-R, Batería de Evaluación de los Procesos Lectores, Revisada (5 ${ }^{a}$ Edición). Madrid: TEA Ediciones.

Cuetos, F.; Ramos, J. L., \& Ruano, E. (2004). PROESC, Evaluación de los procesos de escritura. Madrid: TEA Ediciones.

De Cerrud, A. Bermúdez, L. Barrett, R., Santana, D., \& González, I. (2019).

Estudio de la lectura y escritura de alumnos con discapacidad de la provincia de Coclé. Revista Pinceduc I+D, 2, 48-77.

Hayes, J. R. (1996). A new framework for understanding cognition and affect in writing. In C. Michael Levy \& Sarah Ransdell (Eds.), The Science of Writing (pp. 1-27). Hillsdale, $\mathrm{NJ}$ : Lawrence Erlbaum Associates.

Hayes, J. R., \& Flower, L. (1980). Identifying the organization of writing process. In Lee W. Gregg \& Erwin R. Steinberg (Org.), Cognitive process in writing (pp. 3-30). Hillsdale, NJ: Lawrence Erlbaum Associates.
Hayes, J. R., \& Flower, L. S. (1980). Identifying the organization of writing processes. En L.W. Gregg y E.R. Steinberg (Eds.), Cognitive processes in writing (pp. 3-30). Hillsdale: Lawrence Erlbaum Associates.

Lecuona, Ma. P. (Dir.) (1999). Pensar para escribir. Un programa de enseñanza para la composición escrita. Madrid: CIDE.

Orozco, Y. (2019). Estudio descriptivo de las competencias en lectura y escritura de la población escolar del Instituto Panameño de Habilitación Especial. Revista Pinceduc I+D, 2, 93-102.

Pérez, M., De Acosta, J., \& Rodríguez, A. (2019). Estudio de las competencias lectora y escritora en la población con discapacidad en Darién. Revista Pinceduc I+D, 2, 78-92.

Rodríguez, A. (2019). Estudio sobre alfabetización de la población escolar con discapacidad en Panamá. Revista Pinceduc I+D, 2, 9-47.

Salvador, F. (2008). Psicopedagogía de la lengua escrita. Madrid: EOS.

Salvador, F. (Ed.) (2005). La expresión escrita de alumnos con necesidades educativas especiales. Procesos cognitivos. Málaga: Aljibe.

Salvador, F., \& Gutiérrez, R. (2005). Atención educativa al alumnado con dificultades en la lectura y escritura. Málaga: Aljibe.

Swaby, H. (2019). Estudio de la alfabetización de los alumnos con discapacidades en la provincia de Panamá Oeste. Revista Pinceduc I+D, 2, 103-142.

Treviño, E., Pedroza, H., Pérez, G., Ramírez, P., Ramos, G., \& Treviño, G. (2007). Prácticas docentes para el desarrollo de la comprensión lectora en primaria. Distrito Federal, México: Instituto Nacional para la Evaluación de la Educación. Recuperado de http://www. inee. edu.mx/images/stories/Publicaciones/ Recursosyprocesos/Practicas/Completo/ practicasdocentescompletoa. pdf. 